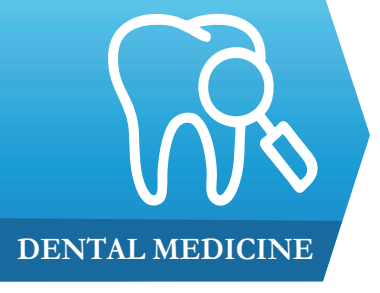

1) Department of Public Health Dentistry, College of Dental Sciences and Hospital, Rau, Indore, India

2) Department of Public Health Dentistry, People's College of Dental Sciences Bhopal, India

3) Department of Oral Medicine and Radiology, College of Dental Sciences and Hospital, Amargadh, District BhavnagarGujarat, India
DOI: $10.15386 /$ cjmed-906

Manuscript received: 03.07.2017 Received in revised form: 20.12.2017 Accepted: 12.01.2018

Address for correspondence: drchole2@rediffmail.com

\title{
Study of oral potentially malignant disorders related to various risk factors amongst the patients attending hospitals in Bhopal, India
}

Swati Balsaraf ${ }^{1}$, Ajay Bhambal ${ }^{2}$, Revant Chole ${ }^{3}$

\begin{abstract}
Background and aims. Oral potentially malignant disorders constitute one of the major oral health problems in India. Few studies have been undertaken on potentially malignant disorders in central India, specifically in Bhopal. Hence the present study was conducted to assess the oral potentially malignant disorders in the patients attending hospitals of Bhopal, India.

Methods. Four hundred subjects diagnosed with oral potentially malignant disorders were included in this study. Detailed information was gathered in a proforma and emphasis was given to the various addictions and clinical findings.

Results. Among the 400 oral potentially malignant disorders diagnosed during the study period, males constituted $78 \%(\mathrm{n}=312)$ and females $22 \%(\mathrm{n}=88)$. The mean age of the patients was $33.50 \pm 13.24$ years for males and $42.60 \pm 14.18$ years for females. The most commonly affected age group was $21-30$ years (37.5\%), followed by $31-40$ years $(25 \%)$ and $41-50$ years $(13 \%) .87 .75 \%$ patients were addicted to chewing of betel nut and/or tobacco and/or betel quid, $15.25 \%$ were addicted to smoking bidi and/or cigarette, $15.25 \%$ were addicted to chewing and smoking, $1 \%$ was addicted to chewing and alcohol, and $0.25 \%$ was addicted to alcohol and smoking.

Conclusion. In this study, an increase in clinical grading of oral potentially malignant disorders was found with severity and duration of addiction habit. In order to decrease the prevalence of these disorders, it is imperative to continue educational campaigns at national and international level to impart education to the public about the risk factors and early signs and symptoms of the oral potentially malignant disorders.
\end{abstract}

Keywords: oral disorders, malignancy potential, oral submucous fibrosis, oral leukoplakia

\section{Introduction}

Oral cavity cancer accounts for approximately $4 \%$ of all malignancies and is a significant worldwide health problem [1]. A wide array of conditions has been implicated in the development of oral cancer. The early detection of cancer is of critical importance because survival rates markedly improve when the oral lesion is identified at an early stage. Features of oral precancerous diseases associated with risk of progression to cancer include color (red, red-white), irregularity (lack of homogeneity), surface texture (granular, verrucous), and location (floor of mouth, ventral or posterolateral border of the tongue) [2].

The World Health Organization (WHO) in 1978 proposed that clinical presentations of the oral cavity that are recognized as precancerous, be classified into 2 groups, as precancerous lesion and precancerous condition. A precancerous lesion is a morphologically altered tissue in which oral cancer is more likely to occur than its apparently normal counterpart. These precancerous lesions include oral leukoplakia (OL), oral erythroplakia, and the palatal lesions of reverse smokers. A precancerous condition is a generalized state associated with significantly increased risk of cancer. The precancerous conditions include oral submucous fibrosis (OSF), oral lichen planus, epidermolysis bullosa, discoid lupus erythematous. At a workshop coordinated by the WHO collaborating centre for oral cancer and precancer in the UK, the term "potentially malignant disorders" was recommended to refer to precancer as it conveys that not all disorders described under this term may transform into cancer [3].

In the Indian subcontinent, the use of smokeless tobacco and betel nut 
in various forms is very popular, which play a role in the development of oral potentially malignant disorder. Tobacco smoking and alcohol abuse are involved in the pathogenesis of oral cavity cancer, and the two agents appear to act synergistically. Although oral leukoplakia and oral sub mucous fibrosis are both high-risk preneoplastic states, the independent and interactive associations between cigarette smoking, alcohol consumption and areca nut chewing have not been well established in these oral diseases. Few studies have been undertaken on oral potentially malignant disorder in central India specifically in Bhopal. Hence the present study was conducted to assess the oral potentially malignant disorders in the patients attending hospital's outward patient department (OPD) of Bhopal.

\section{Materials and methods}

The study is of an observational cross-sectional design. The target population included patients with oral potentially malignant disorder attending dental hospital's OPD with related habits. Madhya Pradesh, as its name implies, is located at the geographic centre of India, with Bhopal as the state capital. Very few studies have been done regarding potentially malignant disorder and their association with tobacco, betel nut chewing and alcohol consumption in Bhopal.

All the dental teaching hospitals were included in the study. The list of all the dental teaching hospitals includes People's College of Dental Sciences and Research Centre, People's Dental Academy, Rama Krishna Dharmarth Foundation (RKDF) Dental College and Research Centre, Bhabha College of Dental Sciences, and Rishiraj College of Dental Sciences and Research Centre, Bhopal, India. The above mentioned are highly regarded dental teaching institutes and hospitals in Bhopal, and are accessible to patients from all socioeconomic groups. Subjects who visited the hospital's Oral Medicine and Radiology department during January 2015 to December 2016 who were having oral potentially malignant disorder on clinical examination were included in this study. All patients were informed about the details of the study and gave their informed consent before starting the study.

Collection of data was done using a proforma which consisted of a questionnaire and clinical examinations. Patients of all age were included in the study. Those who gave a history of usage of tobacco, betel nut, betel leaf and alcohol and having oral premalignant lesions and conditions were included in this study. Patients with oral potentially malignant disorder were identified and details were noted down in the proforma. Patients with any medical problem were excluded from the study.

Emphasis was given to addictions like areca nut, tobacco and alcohol. For all of the chewers and smokers, a detailed history of their chewing and smoking habits was recorded, including daily consumption and duration of practice. For drinkers, information of the frequency of alcohol intake was collected. Recording the data for oral mucosal lesions was based on publications of the World Health Organization criteria. Definition and classification of potentially malignant disorder of oral mucosa were adapted from publication by Warnakulasuriya $\mathrm{S}$ et al. [3]. Detailed clinical examination of each patient was done to assess the site, size and type of lesion. Leukoplakia was diagnosed as a white patch or plaque that cannot be characterized clinically or pathologically as any other disease. Erythroplakia was diagnosed as a bright red velvety plaque or patch which cannot be characterized clinically or pathologically as being due to any other condition. Patients having habit of reverse smoking with significantly more pronounced palatal alterations like erythroleukoplakic were identified as palatal lesions of reverse smoking. Lichen planus was diagnosed as lacelike keratotic mucosal configurations slightly elevated fine whitish lines that produce either a lacelike pattern or a pattern of fine radiating lines; or keratotic change combined with mucosal erythema. Cutaneous lesions appearing red and somewhat scaly patches favoring sun-exposed areas such as the face, chest, back, and extremities with central atrophy, scar formation, and loss of surface pigmentation along with oral mucosal ulcerations combined with keratosis and erythema were identified as discoid lupus erythematosus. Patients having habit of betel nut chewing and complaining of oral burning sensation and ulcerations, and eventual reduced mouth opening with stiffness of the oral mucosa and causing trismus and inability to eat were diagnosed as oral submucous fibrosis. It was classified as: Stage I - Interincisal mouth opening $>3 \mathrm{~cm}$; Stage II - Interincisal mouth opening $=2-3 \mathrm{~cm}$; and Stage III Interincisal mouth opening $<2 \mathrm{~cm}$.

After the provisional diagnosis each patient were referred for biopsy and histopathological examination. After the final diagnosis was established, patients were educated regarding the harmful effects of betel nut, tobacco and alcohol consumption and were referred to the oral medicine and oral surgery specialist for further treatment. Ethical clearance was obtained from the ethical committee People's College of Dental Sciences and Research Centre, Bhopal. The indicator of socioeconomic strata (SES) was recorded according to the Modified Kuppuswamy Scale of 2007 [4]. Data were entered and analyzed using the SPSS software (17.0).

\section{Results \\ Oral potentially malignant disorder and sociodemographic factors}

There were 400 patients diagnosed with potentially malignant disorder in the time period of January 2013 to December 2014. Among the 400 patients diagnosed with oral potentially malignant disorder during the study period, males constituted $78 \%(n=312)$ and females $22 \%(n=88)$. The mean age of the patients was $33.50 \pm 13.24$ years for males and $42.60 \pm 14.18$ years for females. The age 
range of patients was between 12 to 90 years. The most commonly affected age group was $21-30$ years $(37.5 \%)$, followed by $31-40$ years $(25 \%)$ and $41-50$ years $(13 \%)$ (Table I). In terms of religion, Hindus constituted $80.5 \%$ $(\mathrm{n}=322)$ followed by Muslims $17.25 \%(\mathrm{n}=69)$, Sikh $1.75 \%$ $(\mathrm{n}=7)$, Buddhist $0.5 \%(\mathrm{n}=2)$ and Christian $0 \%(\mathrm{n}=0)$.

Based on Modified Kuppuswamy Scale 2007, most of the patients were graduates/post graduates $(49.75 \%)$, based on occupation $38.5 \%$ were unemployed, $28 \%$ had family monthly income in the range of rupees $4894-7322$ and $32.75 \%$ belonged to upper middle and middle lower middle class.

Distribution of patients with oral potentially malignant disorder according to oral habits

$87.75 \%$ patients were addicted to chewing of betel nut and/or tobacco and/or betel quid, $15.25 \%$ were addicted to smoking bidi and/or cigarette, $15.25 \%$ were addicted to chewing and smoking, $1 \%$ was addicted to chewing and alcohol, and $0.25 \%$ was addicted to alcohol and smoking (Table II). Chewing habits was more in graduate and post graduate qualified patients as compared to other educational group and in upper middle class patients.
Distribution of patients according to various oral potentially malignant disorder

Out of 400 oral potentially malignant disorders patients, 39 were having oral leukoplakia, 1 was having erythroplakia, 3 were having palatal lesion of reverse smoking, 342 were having oral submucous fibrosis, 14 were having oral lichen planus and 1 was having discoid lupus erythematosus. Out of 39 oral leukoplakia patients, 34 had homogenous leukoplakia, 4 had speckled leukoplakia and 1 had verrucous leukoplakia. Among the 39 oral leukoplakia lesions, $31(79.48 \%)$ cases were detected in males and 8 $(20.51 \%)$ in females, with a proportion of 1:0.25. The mean age of the patients was $45.19 \pm 13.92$ years for males and $46.62 \pm 13.45$ years for females. The age range of patients was between 20 to 75 years. The most commonly affected age group was $41-50$ years $(35.89 \%)$ followed by $21-30$ years $(23.07 \%)$ and $51-60$ years $(15.38 \%)$. Twenty nine (74.35\%) patients with oral leukoplakia were chewers and $13(33.33 \%)$ were smokers, while 13 (33.33\%) were both chewers and smokers. Majority of patients had the habit for $>5$ years and frequency for $>5$ per day (Table III).

Table I. Age distribution of oral premalignant patients.

\begin{tabular}{|c|c|c|c|c|c|c|c|c|}
\hline $\begin{array}{l}\text { Age group } \\
\text { (Years) }\end{array}$ & $\begin{array}{l}\text { Leuko } \\
\text {-plakia (\%) }\end{array}$ & $\begin{array}{l}\text { Erythro } \\
\text {-plakia } \\
(\%)\end{array}$ & $\begin{array}{l}\text { Palatal Lesion of } \\
\text { reverse smoking } \\
(\%)\end{array}$ & $\begin{array}{l}\text { Oral Sub } \\
\text { mucous } \\
\text { Fibrosis (\%) }\end{array}$ & $\begin{array}{l}\text { Lichen } \\
\text { Planus (\%) }\end{array}$ & $\begin{array}{l}\text { Actinic } \\
\text { Keratosis } \\
(\%) \\
\end{array}$ & $\begin{array}{l}\text { Discoid Lupus } \\
\text { Erythe - matosus } \\
(\%)\end{array}$ & Total (\%) \\
\hline 0-10 & $00(0)$ & $00(0)$ & $00(0)$ & $00(0)$ & $00(0)$ & $00(0)$ & $00(0)$ & $00(0)$ \\
\hline $11-20$ & $01(2.5)$ & $00(0)$ & $00(0)$ & $36(10.5)$ & $02(14.28)$ & $00(0)$ & $00(0)$ & $039(9.75)$ \\
\hline $21-30$ & $09(23.07)$ & $00(0)$ & $00(0)$ & $137(40.05)$ & $04(28.5)$ & $00(0)$ & $00(0)$ & $150(37.5)$ \\
\hline $31-40$ & $04(10.25)$ & $01(100)$ & $01(33.3)$ & 091 (26.6) & $03(21.4)$ & $00(0)$ & $00(0)$ & $100(25)$ \\
\hline $41-50$ & $14(35.8)$ & $00(0)$ & $01(33.3)$ & $035(10.23)$ & $02(14.2)$ & $00(0)$ & $00(0)$ & $052(13)$ \\
\hline $51-60$ & $06(15.3)$ & $00(0)$ & $01(33.3)$ & $025(7.3)$ & $03(21.4)$ & $00(0)$ & $01(100)$ & $036(9.0)$ \\
\hline $61-70$ & $04(10.25)$ & $00(0)$ & $00(0)$ & $015(4.38)$ & $00(0)$ & $00(0)$ & $00(0)$ & $019(4.75)$ \\
\hline $71-80$ & $01(2.5)$ & $00(0)$ & $00(0)$ & $002(0.5)$ & $00(0)$ & $00(0)$ & $00(0)$ & $003(0.75)$ \\
\hline $81-90$ & $00(0)$ & $00(0)$ & $00(0)$ & $001(0.2)$ & $00(0)$ & $00(0)$ & $00(0)$ & $001(0.25)$ \\
\hline Total & $39(100)$ & 01 (100) & 03 (100) & $342(100)$ & $14(100)$ & $00(0)$ & $01(100)$ & $400(100)$ \\
\hline
\end{tabular}

Table II. Distribution of patients according to personal habits.

\begin{tabular}{llll}
\hline & Personal habits & Number of patients (\%) & Total (\%) \\
\multirow{2}{*}{ Chewing } & Betel nut & $76(19)$ & \\
& Tobacco + Slaked lime & $70(17.5)$ & $\mathbf{3 5 1}(\mathbf{8 7 . 7 5 )}$ \\
& Betel nut + Tobacco & $183(45.75)$ & \\
Smoking & Betel leaf + Betel nut + Tobacco & $22(5.5)$ & \\
Alcohol & Bidi / Cigarette/reverse smoking & $25(6.2) / 32(8) / 04(1)$ & $\mathbf{6 1}(\mathbf{1 5 . 2 5 )}$ \\
& & $00(0)$ & \\
Combinations & Chewing + Smoking & $61(15.25)$ & $\mathbf{6 6}(\mathbf{1 6 . 5 )}$ \\
& Alcohol + Chewing & $04(1)$ &
\end{tabular}

Table III. Distribution of patients with oral leukoplakia according to duration of chewing habits and clinical staging.

\begin{tabular}{|c|c|c|c|c|c|c|}
\hline Duration (in years) & Homogenous (\%) & Speckled (\%) & Verrucous (\%) & Proliferative verrucous (\%) & Total (\%) & p-value \\
\hline$\geq 5$ & $14(41.1)$ & $00(0)$ & $00(0)$ & $00(0)$ & $14(35.8)$ & \multirow{6}{*}{$\mathrm{P}<0.05$} \\
\hline $6-10$ & $08(23.5)$ & $01(25)$ & $00(0)$ & $00(0)$ & 09 (23.07) & \\
\hline $11-15$ & $05(14.7)$ & $00(00)$ & $00(0)$ & $00(0)$ & $05(12.8)$ & \\
\hline $16-20$ & $05(14.7)$ & $02(50)$ & $00(0)$ & $00(0)$ & 07 (17.9) & \\
\hline$>20$ & $02(5.8)$ & $01(25)$ & $01(100)$ & $00(0)$ & $04(10.25)$ & \\
\hline Total & $34(100)$ & $04(100)$ & $01(100)$ & $00(0)$ & $39(100)$ & \\
\hline
\end{tabular}


The buccal mucosa was the site most affected (n $=35)$, followed by the tongue $(n=3)$, palate $(n=1)$ and labial mucosa $(n=1)$. Out of 342 OSF patients, 255 were in Stage 1, 76 were in Stage 2 and 11 were in Stage 3. Among the 342 OSF patients, $267(78.07 \%)$ cases were detected in males and $75(21.93 \%)$ in females, with a proportion of 1:0.28. The mean age of the patients was $32.31 \pm 12.58$ years for males and $41.90 \pm 15.43$ years for females. The age range of patients was between 12 to 80 years. The most commonly affected age group was $21-30$ years $(40.05 \%)$ followed by $31-40$ years $(26.60 \%), 11-20$ years $(10.52 \%)$ and $41-50$ years $(10.23 \%)$. Two hundred eighty one $(82.16 \%)$ patients were chewers, $39(11.40 \%)$ were smokers, 4 consumed alcoholic beverages $(1.16 \%)$, while $39(11.40 \%)$ were both chewers and smokers, 4 were both chewers and alcoholic (1.16\%) and 1 was both smoker and alcoholic $(0.29 \%)$. Majority of patients had the habit for $>$ 5 years and frequency for $>5$ per day $(\mathrm{p}<0.05)($ Table IV).

Table IV. Distribution of patients with oral submucous fibrosis according to duration of chewing habits and clinical staging.

\begin{tabular}{l|l|l|l|l|l}
$\begin{array}{l}\text { Duration } \\
\text { (in years) }\end{array}$ & STAGE 1 & STAGE 2 & STAGE 3 & Total & p-value \\
$\mathbf{2 5}$ & $127(49.80)$ & $36(47.3)$ & $04(36.3)$ & $\mathbf{1 6 7 ( 4 8 . 8 )}$ & \\
$\mathbf{6 - 1 0}$ & $69(27.05)$ & $27(35.5)$ & $04(36.3)$ & $\mathbf{1 0 0 ( 2 9 . 2 3 )}$ & \\
$\mathbf{1 1 - 1 5}$ & $26(10.1)$ & $10(13.1)$ & $02(18.1)$ & $\mathbf{3 8}(\mathbf{1 1 . 1})$ & $\mathrm{P}<0.05$ \\
$\mathbf{1 6 - \mathbf { 2 0 }}$ & $17(6.6)$ & $01(1.3)$ & $00(00)$ & $\mathbf{1 8 ( 5 . 2 6 )}$ & \\
$\mathbf{2 \mathbf { 2 0 }}$ & $16(6.2)$ & $02(2.6)$ & $01(9.09)$ & $\mathbf{1 9 ( 5 . 5 )}$ & \\
Total & $255(100)$ & $76(100)$ & $11(100)$ & $\mathbf{3 4 2 ( 1 0 0 )}$ &
\end{tabular}

The buccal mucosa was the site most affected $(\mathrm{n}=327$, $95.61 \%)$, followed by the palate $(\mathrm{n}=161,47.07 \%)$, retromolar region $(n=112,32.74 \%)$, floor of the mouth $(n=60,17.54 \%)$ and labial mucosa $(\mathrm{n}=46,13.45 \%)$. Burning sensation in oral mucosa was the most frequent symptom $(92.69 \%)$, while recurrent ulcerations were present in $116(33.92 \%)$. One male patient was diagnosed as erythroplakia in the age group of 31-40 years. The site involved was buccal mucosa. Out of three cases of palatal lesions of reverse smoking all were males and one patient each in the age group of 31-40, 41-50 and 51-60 years. No case was detected for actinic keratosis. One case of discoid lupus erythematosus was seen in a male patient in the age group of 51-60 years (Table I). Out of 14 oral lichen planus patients, the reticular form was observed in $8(57.14 \%)$ patients and the erosive form was observed in $06(42.85 \%)$ cases. Among the 14 oral lichen planus patients, $9(64.28 \%)$ cases were detected in males and $5(35.71 \%)$ in females, with a proportion of 1:0.3. The mean age of the patients was $33.40 \pm 14.36$ years for males and $46.60 \pm 9.81$ years for females. The age range of patients was between 19 to 59 years. The most commonly affected age group was 2130 years $(28.57 \%)$ followed by $31-40$ years $(21.40 \%), 51-60$ years $(21.40 \%)$ and $41-50$ years $(14.28 \%)$. Five $(35.71 \%)$ patients with the disease were smokers and none consumed alcoholic beverages. The buccal mucosa was the site most affected $(n=12)$, followed by labial mucosa $(n=2)$. Burning sensation in oral mucosa was the most frequent symptom (71.42\%). Among patients with painful symptoms, $83.33 \%$ had the erosive form.

\section{Discussion}

This study is of an observational cross-sectional design. The target population included patients with oral potentially malignant disorders attending dental hospital's OPD with related habits. All the dental colleges and hospitals in the central Indian city of Bhopal were included in the study. 400 patients with oral potentially malignant disorders and related habits were included in the study in the time period of January 2015 to December 2016.

\section{Sociodemographic factors}

In this study out of 400 patients examined, males constituted $78 \%(n=312)$ and females $22 \%(n=88)$. The maximum patients were in the age group of 21-30 years (37.5\%), followed by $31-40$ years $(25 \%)$ and $41-50$ years $(13 \%)$. The results are in line with a study [5] in which there were more males $(63.75 \%)$ in the study population than females $(36.25 \%) .17 .15 \%$ of the study participants were in the age group of 13 to 20 years, $38.13 \%$ were in the age group of 21 to 30 years, $21.47 \%$ were in the age group of 31 to 40 years and the remaining $23.25 \%$ were in the age group of 41 to 84 years. The results are also in line with a study in which maximum patients were in the age group of 16-30 years followed by 31-45 years and 4660 years age group [6]. It could be attributed to the fact when tobacco and betel nut use among are out-of-school, earning, less educated and live in rural areas are more likely to use tobacco and start during the preteen years.

In our study the mean age of the patients was 33.50 \pm 13.24 years for males and $42.60 \pm 14.18$ years for females. The results were similar to a study done which stated that most affected patients are middle-aged or elderly men [7]. In our study out of 400, maximum patients were of oral submucous fibrosis. This could be attributed to the fact that that gutkha/ betel nut is available at roadside stands, tea stalls, cigarette shops and grocery stores in India. Above all it is conveniently priced and within easy reaches. In our study the premalignant lesions and conditions were most commonly found on the buccal mucosa, palate, floor of the mouth, gingival and retromolar region, similar to the findings of a study [7].

When the patients of leukoplakia were divided according to their habits it was found that 27 out of 39 cases of leukoplakia that is $69.23 \%$ were smokeless tobacco users followed by smokers (13.3\%) and the results were found to be statistically significant $(p<0.05)$. The results were not similar to the findings of a study [8] who have described tobacco induced changes in oral leukoplakic epithelium in groups of Danish and Indian Leukoplakia patients, but these appeared to be associated chiefly with the use of pipes, snuff and hooklis (a short stemmed clay pipe used in India). In our study the most commonly affected age group for leukoplakia was 41-50 years (35.89\%).The 
findings are not in line with a longitudinal study in which the age distribution of leukoplakia showed that the highest frequency occurred in the age group 51-60 years [9].

Oral submucous fibrosis is a potentially malignant disease of oral cavity and is most commonly found in Asian countries. It constitutes one of the major oral health problems in countries like India. In this study, out of 342 OSF patients $267(78.07 \%)$ were males and $75(21.93 \%)$ females with a male to female ratio of 1:0.28. Maximum, 137 were in the 21-30 years age group. In a study similar results were found in central India, in which majority of OSF patients were in the younger age group ( $<30$ years) with a male to female ratio of 5:1 [10]. Our results are not in line with other study in which only $17.3 \%$ were betel nut chewers [11].

In case of oral lichen planus the clinical characteristics of the patients studied here were similar to those reported in the literature, although some differences were observed. A predominance of OL among male patients was observed in the present study, not in agreement with other reports [12-14]. A predominance of OL in the second and fifth decades of life was observed in the present study, in agreement with a study on British patients [14]. There are no literature data indicating an elevated prevalence of smoking or alcohol consumption among patients with OL compared to the general population, a finding also observed in the present study.

The buccal mucosa was the site most affected, followed by the labial mucosa in agreement with other reports $[12,14]$. No extraoral manifestations were observed in the patients studied. According to the literature, $50 \%$ of all patients with lichen planus simultaneously present skin and oral lesions, whereas $25 \%$ present only oral lesions [15]. In contrast, other studies have reported cutaneous involvement in less than $17 \%$ of patients with OL $[12,15]$. The reticular form was the most frequent, followed by the erosive form. Burning sensation in oral mucosa was the most frequent symptom (71.42\%). Among patients with painful symptoms, $83.33 \%$ had the erosive form. Similar results have been reported in previous studies $[13,16]$.

In the present study only 1 case of erythroplakia, 3 of palatal lesion of reverse smoking and 1 case of discoid lupus erythematosus were observed due to which it was not possible to compare them with the existing literature in a scientifically meaningful manner.

\section{Risk factors}

In our study when patients were distributed according to their habits it was found that out of 400 patients $68.75 \%$ of them had the chewing habit, $15.25 \%$ had smoking habit and $15.25 \%$ had both chewing and smoking habit. The above two findings that is habit of chewing and smoking are in line with the findings of a study which showed that $55.5 \%$ of patients had tobacco chewing habit followed by smoking habit $44.4 \%$ [6]. But the patients who had both the habits were more in number than smoking alone which is not in line with our study.
In our study when the patients were distributed according to their education and habits it was found that maximum patients were graduates and postgraduates followed by undergraduates and then illiterate, the results are not in line with a study who found that maximum patients with the habit of tobacco chewing were undergraduates followed by uneducated and then graduates [6]. Another study found that, smoking, chewing tobacco alone or included in pan are the causative factors for leukoplakia [17]. Similar findings were found in our study.

In our study most of the patients with oral submucous fibrosis had the habit of chewing less than or equal to five years and less than or equal to 5 times per day. The results are similar to a study done in Patna, India [18]. The OSF cases used gutkha and other products 2-10 pouches per day and kept in the mouth for 2-10 minutes and they were using since 2-4 years. Most of the OSF cases kept gutkha in the buccal vestibule or they chewed and swallowed it, only a small number of patients chewed and spitted it out. It was also observed that OSF developed on one side of the buccal vestibule where they kept the chew and other side was normal.

This study found that betel quid chewing was the principal cause of OL and OSF. The risks increased with the duration and frequency of the habit, as previously shown in India, Pakistan, Taiwan and Mainland China $[19,20]$. In our study majority of patients had habit for $>5$ years and frequency for $>5$ per day. The data indicated that even a relatively short exposure is sufficient to induce leukoplakia or submucous fibrosis, as previously suggested [19,21]. Arecoline, the most abundant alkaloid in areca nut, has been observed experimentally to stimulate collagen synthesis by fibroblasts in vitro [22]. Studies of human buccal fibroblasts found that arecoline was not only cytotoxic but stimulated double-stranded polynucleic acid synthesis; both might act synergistically on the pathogenesis of OSF as well as oral cancer [23].

Oral leukoplakia, erythroplakia, lichen planus and OSF are clinically distinct precancerous lesions that precede the development of oral cancer. A study in Taiwan showed that the risk of OSF at each exposure level of betel quid chewing was stronger than those of OL, although the difference was not large enough to reject the null [24]. We also found that mainly younger patients had OSF compared with mainly older patients with OL. The fact that OSF patients started betel quid chewing at a younger age than OL patients and chewed more quid per day may partly explain the age differences between the two diseases.

In our study no correlation was found between the severity of OSF and betel nut, tobacco and alcohol consumption $(\mathrm{p}>0.05)$. In an Indian study $38(15.8 \%)$ patients were addicted to betel quid with areca nut and tobacco, 14 (5.9\%) males were addicted to smoking alone, and only $2(0.8 \%)$ males were habituated to alcohol, but no consistent correlation was found between the OSF and smoking/alcohol consumption. Clinically, trismus is an 
important symptom of OSF. In this study, 255 (74.56\%) patients had stage I trismus, $76(22.22 \%)$ had stage II and $11(3.21 \%)$ had stage III trismus [25]. Another study reported the trismus was the chief complaint in $90.8 \%$ of their patients [26]. Previous research reported that maximum patients in their study had stage II trismus [25], while another study reported that maximum patients of OSF had stage III trismus [10].

On the correlation of addiction habit and clinical findings, maximum patients had clinical grade I OSF and took tobacco products for $>5$ years with frequency $>5$ times per day. Daily consumption of betel quid was more significant that the total duration of the habit. In one study maximum patients had histopathological grade III OSF and took tobacco products for 8-10 years or more with high frequency (7-10 times per day) followed by histopathological grades II and I [25].

Oral potentially malignant disorders converting to oral cancer has been one of the 10 leading causes of cancer deaths in India. Tobacco products are today part of the modern urban as well as rural life style. The widespread habit of chewing betel nut and tobacco is a major risk factor of oral potentially malignant disorders, especially in the younger age group. In this study, an increase in clinical grading was found with severity and duration of addiction habit. In order to decrease the prevalence of these disorders, it is imperative to continue educational campaigns at national and international level, to impart education to the public about the risk factors and early signs and symptoms of the oral potentially malignant disorders.

\section{References}

1. Epstein JB, Gorsky M, Cabay RJ, Day T, Gonsalves W. Screening for and diagnosis of oral premalignant lesions and oropharyngeal squamous cell carcinoma: role of primary care physicians. Can Fam Physician. 2008;54:870-875.

2. Zhang L, Cheung KJ Jr, Lam WL, Cheng X, Poh C, Priddy $\mathrm{R}$, et al. Increased genetic damage in oral leukoplakia from high risk sites: potential impact on staging and clinical management. Cancer. 2001;91:2148-2155.

3. Warnakulasuriya S, Johnson NW, van der Waal I. Nomenclature and classification of potentially malignant disorders of the oral mucosa. J Oral Pathol Med. 2007;36:575-580.

4. Kumar N, Shekhar C, Kumar P, Kundu AS. Kuppuswamy's socioeconomic status scale-updating for 2007. Indian J Pediatr. 2007;74:1131-1132.

5. Saraswathi TR, Ranganathan K, Shanmugam S, Sowmya R, Narasimhan PD, Gunaseelan R. Prevalence of oral lesions in relation to habits: Cross-sectional study in South India. Indian J Dent Res. 2006;17:121-125.

6. Patel PS, Raval GN, Patel DD, Sainger RN, Shah MH, Shah JS, et al. A Study of Various Sociodemographic Factors and Plasma Vitamin Levels in Oral and Pharyngeal Cancer in Gujarat, India. Asian Pac J Cancer Prev. 2001;2:215-224.

7. Napier SS, Speight PM. Natural history of potentially malignant oral lesions and conditions: an overview of the literature. J Oral Pathol Med. 2008;37:1-10.
8. Pindborg JJ, Reibel J, Roed-Peterson B, Mehta FS. Tobaccoinduced changes in oral leukoplakic epithelium. Cancer. 1980;45:2330-2336.

9. Bánóczy J, Sugár L. Longitudinal studies in oral leukoplakias. J Oral Pathol Med. 1972;1:265-272.

10. Hazarey VK, Erlewad DM, Mundhe KA, Ughade SN. Oral submucous fibrosis: study of 1000 cases from central India. J Oral Pathol Med. 2007;36:12-17.

11. Reichart PA, Mohr U, Srisuwan S, Geerlings H, Theetranont C, Kangwanpong T. Precancerous and other oral mucosal lesions related to chewing, smoking and drinking habits in Thailand. Community Dent Oral Epidemiol. 1987;15:152-160.

12. Pakfetrat A, Javadzadeh-Bolouri A, Basir-Shabestari S, Falaki F. Oral Lichen Planus: a retrospective study of 420 Iranian patients. Med Oral Patol Oral Cir Bucal. 2009;14(7):E315-E318. 13. Xue JL, Fan MW, Wang SZ, Chen XM, Li Y, Wang L. A clinical study of 674 patients with oral lichen planus in China. J Oral Pathol Med. 2005;34:467-472.

14. Ingafou M, Leao JC, Porter SR, Scully C. Oral lichen planus: a retrospective study of 690 British patients. Oral Dis. 2006;12:463468.

15. Scully C, el-Kom M. Lichen planus: review and update on pathogenesis. J Oral Pathol. 1985;14:431-458.

16. Gorsky M, Raviv M, Moskona D, Laufer M, Bodner L. Clinical characteristics and treatment of patients with oral lichen planus in Israel. Oral Surg Oral Med Oral Pathol Oral Radiol Endod. 1996;82:644-649.

17. Pindborg JJ, Renstrup G, Poulsen HE, Silverman S Jr. Studies in oral leukoplakias. v. clinical and histologic signs of malignancy. Acta Odontol Scand. 1963;21:407-414.

18. Ahmad MS, Ali SA, Ali AS, Chaubey KK. Epidemiological and etiological study of oral submucous fibrosis among gutkha chewers of Patna, Bihar, India. J Indian Soc Pedod Prev Dent. 2006;24:84-89.

19. Maher R, Lee AJ, Warnakulasuriya KA, Lewis JA, Johnson NW. Role of areca nut in the causation of oral submucous fibrosis: a case-control study in Pakistan. J Oral Pathol Med. 1994;23:65-69. 20. Shiu MN, Chen TH, Chang SH, Hahn LJ. Risk factors for leukoplakia and malignant transformation to oral carcinoma: a leukoplakia cohort in Taiwan. Br J Cancer. 2000;82:1871-1874.

21. Seedat HA, van Wyk CW. Betel chewing and dietary habits of chewers without and with oral submucous fibrosis and with concomitant oral cancer. S Afr Med J. 1988;74:572-575.

22. Canniff JP, Harvey W. The aetiology of oral submucous fibrosis: the stimulation of collagen synthesis by extracts of areca nut. Int J Oral Surg. 1981;10(Suppl 1):163-167.

23. Chang YC, Tai KW, Cheng MH, Chou LS, Chou MY. Cytotoxic and non-genotoxic effects of arecoline on human buccal fibroblasts in vitro. J Oral Pathol Med. 1998;27(2):68-71. 24. Lee CH, Ko YC, Huang HL, Chao YY, Tsai CC, Shieh TY, et al. The precancer risk of betel quid chewing, tobacco use and alcohol consumption in oral leukoplakia and oral submucous fibrosis in southern Taiwan. Br J Cancer. 2003;88:366-372.

25. Pandya S, Chaudhary AK, Singh M, Singh M, Mehrotra R. Correlation of histopathological diagnosis with habits and clinical findings in oral submucous fibrosis. Head Neck Oncol. 2009;1:10. 26. Chiu CJ, Lee WC, Chiang CP, Hahn LJ, Kuo YS, Chen CJ. A scoring system for the early detection of oral submucous fibrosis based on a self-administered questionnaires. J Public Health Dent. 2002;62:28-31. 